\title{
Design and Development of Methanol Sensing MEMS Sensor for DMFC Technology
}

\author{
Muthuraja Soundrapandian ${ }^{1 *}$,Subramaniam Chittur Krishnaswamy ${ }^{2}$, \\ ${ }^{1}$ School of Electronics Engineering, VIT, Vellore, 632014 India \\ ${ }^{2}$ School of Advanced Sciences, VIT, Vellore, 632014, India
}

\begin{abstract}
The sensors can be developed based on ampherometric principle using Direct Methanol Fuel Cell (DMFC) technology. The synthesis of cost effective electrocatalyst materials for improved oxygen reduction reaction (ORR) and preparing electrodes by using suitable methods to reduce the cost of the sensing electrode is the major objective of the present work. Pt-Sn alloy exhibits high sensitivity in ORR among other Pt alloys and hence will be used as the ORR catalysts. For various concentration of methanol at different temperature, the current density of the chemically synthesised and characterized $\mathrm{Pt}-\mathrm{Sn} / \mathrm{C}$ was analysed. The accuracy will be determined by the detection of electric current or changes in electric current to design the sensor. To analyse the sensor accuracy, we have used passive mode design protocol in COMSOL Multiphysics. From the constructed cell of $1.0 \mathrm{~cm}$ cell area, we can optimize the overall power density and hence the sensitivity of the sensor by the modification of the cell parameters and interfacing it with Darcy's law of fluidic flow through porous electrode medium. The micro electro mechanical systems (MEMS) technology was used for the design and fabrication of sensor with the electrochemical inputs from a standard DMFC single cell arrangement. The cell structure has been fabricated using a 3D printing technology and the output of the cell was optimized for ampherometric detection. The sensor with microfludic interconnects were fabricated by MEMS technology. The sensor response characteristics were studied and will be presented.
\end{abstract}

\section{Introduction}

The direct methanol fuel cell (DMFC) has promising applications ranging from low power electronic devices to mid-size generators for the automotive domain and for large stationary power systems [1]. In this technology there are several key technical challenges like low rate of methanol oxidation kinetics, methanol crossover, water crossover and thermal management [2]. In this work, the passive mode of design using COMSOL Multiphysics simulation tool was used to provide certain solution for the limitations of DMFC technology. By varying the design parameters we can optimize the power density and sensitivity of the cell $[3,4,5]$. The cell consists of combined seven layers from Graphite Anode inlet to Graphite cathode inlet which are assigned to the different interfaces and its properties. [6] Figure 1 presents the schematic of the cell design configuration.

\footnotetext{
*Corresponding author : muthurajas@vit.ac.in
} 


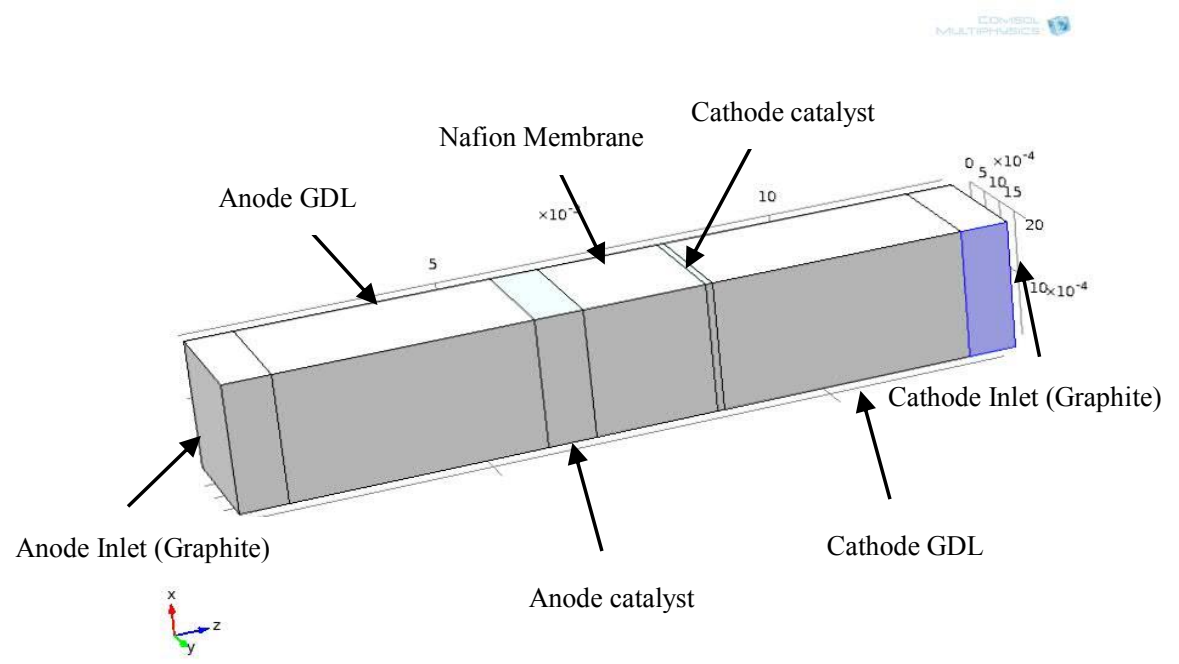

Fig.1. Schematic of the test cell design configuration

The area of the designed cell is $1 \mathrm{~cm}^{2}$. The thickness of anode catalyst layer is taken as $0.2 \mathrm{~cm}$ where the cathode catalyst layer thickness is $0.01 \mathrm{~cm}$. Nafion (117) membrane is chosen as selective proton exchange membrane with 0.178 microns thickness. The carbon matrix based cathode gas diffusion layer (GDL) has standard permeability value of $1.0^{-13} \mathrm{~m}^{2}$. This is used to allow $\mathrm{O}_{2}$ molecules towards cathode catalyst layer to perform cathodic reaction [7]. Anode and cathode inlet pressure was set as 1.1 atmospheres. The cell voltage was assigned as $1.21 \mathrm{~V}$ and the cell temperature, $300 \mathrm{~K}$. The constant anode flow rate of the concentrated methanol is $1 \mathrm{cc} / \mathrm{min}$ where the flow rate of cathode with oxygen is 100 $\mathrm{cc} / \mathrm{min}$. The cell is fabricated with $\mathrm{Pt}$ as catalyst for both anode and cathode electrodes Different concentrations from $0.5 \mathrm{M}, 1 \mathrm{M}, 2 \mathrm{M}$ and $3 \mathrm{M}$ of methanol was fed in to the cell through the anode inlet. The experiment was repeated with the $0.5 \mathrm{M}$ methanol at $375 \mathrm{~K}$ to analyze the influence of the temperature in the cell. We have used pure oxygen for the cathodic reaction [8]. The current density obtained for the active cell area is shown in figure 2a. [5]

The same method was repeated with the Pt alloys like Pt-Ru, Pt-Fe, Pt-Sn and Pt-Mo as anode catalysts where the methanol concentration was $1 \mathrm{M}$ and cell temperature, $300 \mathrm{~K}$. The polarization curves shows improved in the current density when compared with pure $\mathrm{Pt}$ catalyst [9]. The Pt alloys exhibiting high oxygen reduction reaction, ORR and thus producing the better current densities as compare with pure Pt catalysts [10,11]. Among these four alloy compounds, $\mathrm{Pt}-\mathrm{Sn}$ is exhibiting high catalytic reactivity and producing the maximum current density around $0.57 \mathrm{~A} / \mathrm{cm}^{2}$. This is presented in Figure $2 \mathrm{~b}$. 
Current density (mA/Cm2)

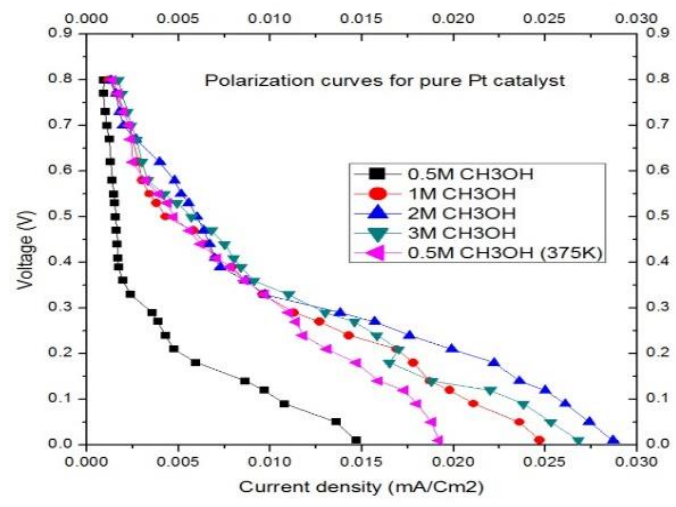

Fig.2.a. Polarization curve for Pt electrodes

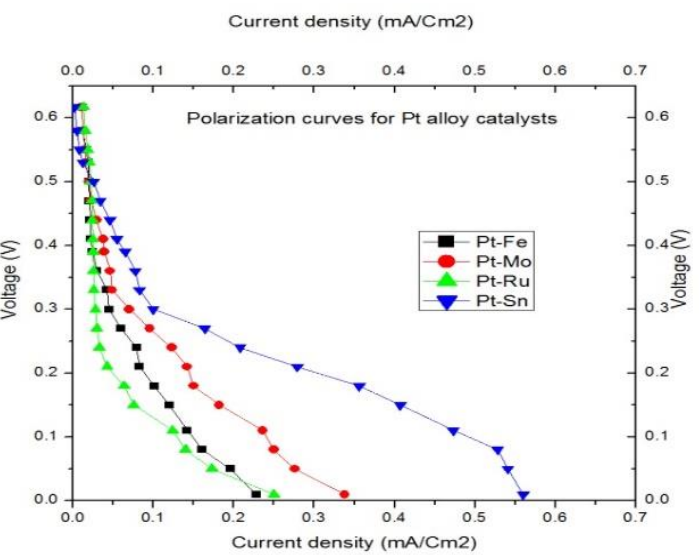

Fig.2.b. Polarization curve for Pt-alloy catalysts

\section{Synthesis and characterization of Pt-Sn/C}

As per the simulation analysis, $\mathrm{Pt}-\mathrm{Sn} / \mathrm{C}$ exhibits high current density as compared with other Pt alloys (12, 13 and 14). The microwave assisted synthesis technique of Pt-Sn/C alloy was used. Catalysts with nominal composition Pt75Sn25/C and 40 wt. \% metal loading were prepared by microwave-assisted heating and thermal decomposition of polymeric precursors, in order to obtain particles with nanometric dimensions. (15).
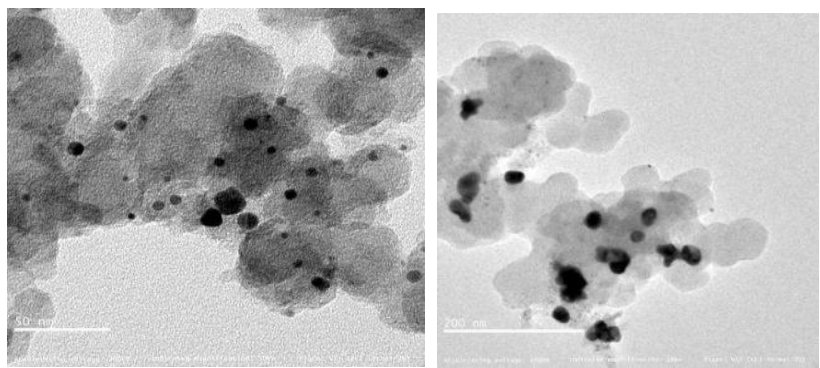

Fig.3. HRTEM images of Pt-Sn/C

Figure 3 presents the HRTEM image of the Pt-Sn/C catalysts. The nano particles of Pt-Sn catalysts were homogeneously distributed on the Vulcan XC carbon support. The nano particles have an average diameter of around $4.5 \mathrm{~nm}$.

\section{Sensor testing}

The MEA was prepared for the synthesized Pt-Sn/C catalyst by the standard methodology and kept in a single cell test assembly for the sensor testing. The current voltage performance of the cell was analysed for the different temperatures by keeping the methanol and oxygen 
flow rate as constant as $3 \mathrm{ml} / \mathrm{min} \& 100 \mathrm{cc} / \mathrm{min}$ respectively. For the lower operating temperature of $650 \mathrm{C}$, from Figure 4 (a), we observe that in the voltage range $0.4 \mathrm{~V}$ to $0.2 \mathrm{~V}$, the change in current was $0.85 \mathrm{~A}$. This is higher than that observed at $900 \mathrm{C}$. This is presented in Figure 4(b). We can say that $\mathrm{Pt}-\mathrm{Sn} / \mathrm{C}$ is exhibiting higher reactivity and producing maximum current response at lower temperature. $[16,17]$

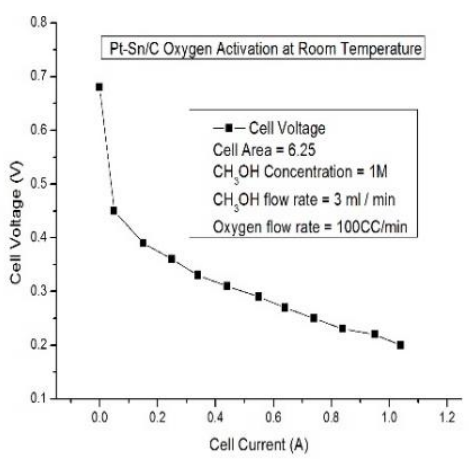

Fig.4.a. Polarization curve using Pt-Sn catalysts at 300K

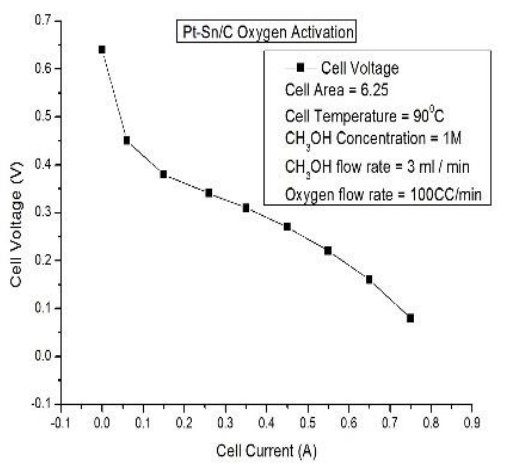

Fig.4.b. Polarization curve using Pt-Sn catalysts at $390 \mathrm{~K}$

\section{MEMS based cell structure}

Solidworks, AutoCAD simulation tool was used to design the MEMS based prototype with microfluidic channels of $1 \mathrm{~mm}$. This is presented in Figure 5(a). The interconnection dimensions of fluidic channel as indicated in Figure5(a) was fabricated on carbon fiber matrix [18].

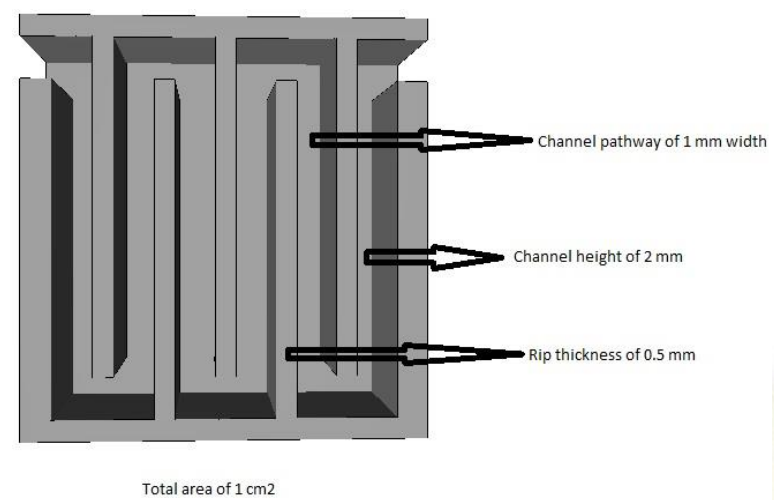

Fig.5.a. Cell structure Solid works profile

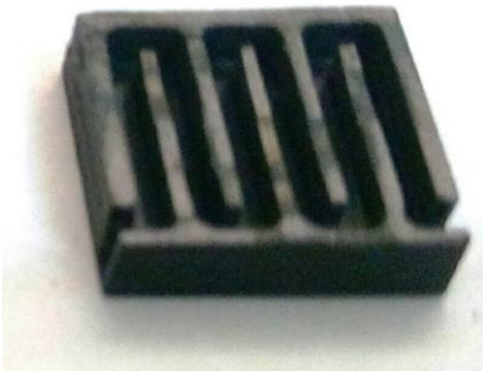

Fig.5.b. Cell Structure 3-D printing

3D printing technology (Stratasys, F123, Objet Eden260VS) has been used for the production of the prototype. $[19,20]$ This is shown in figure 5(b). The work focused on the deposition of selected electrode materials on the fabricated prototype chip to analyze the cell response for different methanol concentrations. 


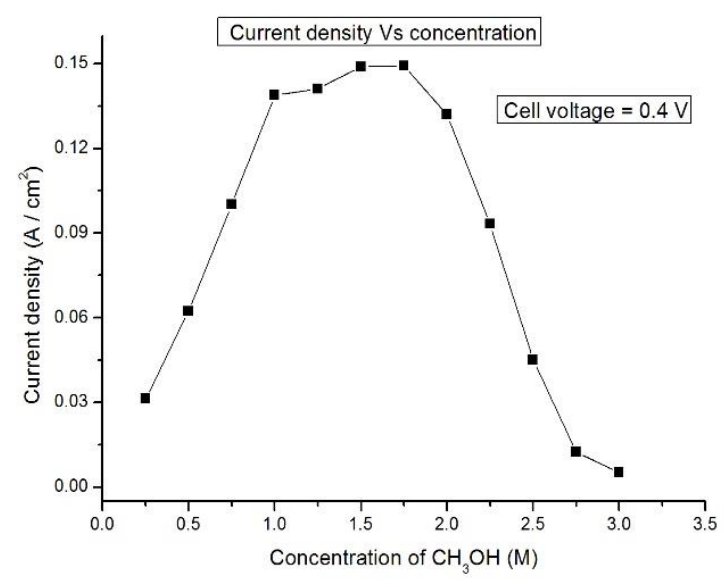

Fig.6. Current density as a function of methanol concentration using Pt-Sn/C catalysts.

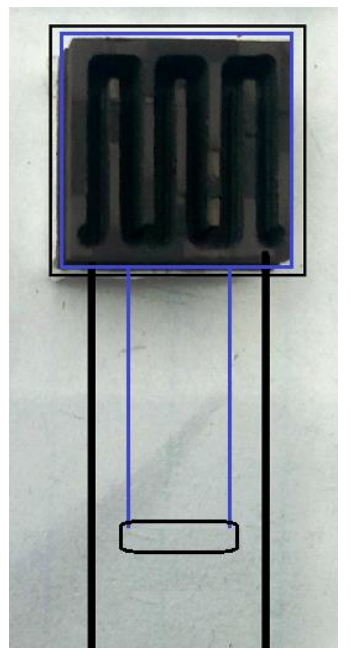

Fig.7. Schematic of sensor chip

The fabricated prototype of interconnected microfluidic channel was embedded with Perspex sheets as shown in Figure 7. The integration of suitable electronic circuits with the sheets to design the miniaturized compact methanol sensor chip is the present work to be done and the results will be presented.

\section{Conclusion}

In this article, the methanol sensing capability of the $\mathrm{Pt}-\mathrm{Sn} / \mathrm{C}$ has been studied. The results from COMSOL Multiphysics simulation tool was analysed in order to improve the sensitivity of the sensor. We find that $\mathrm{Pt}-\mathrm{Sn} / \mathrm{C}$ exhibits excellent sensitivity at lower temperature and hence is a suitable material for this devise. The fabrication of MEMS based chip has been 
done on conducting carbon fiber matrix and using this prototype the sensor studies and characteristics will be evaluated.

The authors sincerely acknowledge the Chancellor of VIT University, Vellore, Tamilnadu, India for the constant support provided to carry out this work.

\section{References}

1. W. Liu, and C. Wang, Journal of The Electrochemical Society, 154,3,B352-B361, (2007).

2. W. J. Yang, H.Y. Wang, Y.B. Kim, International journal of Hydrogen Energy, 39, 94309439, (2014).

3. W. W. Yang, T.S. Zhao, C. Xu, Electrochimica Acta 53, 853-862, (2007).

4. Edmund. F. Dickinson, Henrik Ekström, Ed Fontes, Electrochemistry Communications 40, 71-74, (2014).

5. W.W. Yang, T.S. Zhao, C. Xu, Electrochimica Acta 53, 853-862, (2007).

6. K. Subramaniam Chittur, S. Muthuraja, MRS Online Proceedings Library Archive, Volume 1774, 2015, pp 41-50, doi: 10.1557/opl.2015.746, Published online by Cambridge University Press 11, (2015).

7. Muthuraja Soundrapandian, Subramaniam Chittur Krishnaswamy, ECS Transactions, 75 (17) 85-94 (2016).

8. Abhay Kulkarni, Xia Wang, ECS Transactions, 33 (21) 25-37 (2011).

9. Wonseok Yoona and Xinyu Huang, Journal of The Electrochemical Society, 157,5(B680-B690), (2010).

10. E. U. Ubong, Z. Shi,b, X. Wang, Journal of The Electrochemical Society, 156,10,(B1276-B1282), (2009).

11. Benjamin. E. McNealy and Joshua, Hertz, ECS Transactions, 50 (2) 137-145 (2012).

12. William B. Zimmerman, Chemical Engineering Science 66, 1412-1425, (2011).

13. Matteo Scaramuzza, Alberto Ferrario, Elisabett Pasqualotto, Alessandro De, Toni, Procedia Chemistry 6, 69 - 78, (2012).

14. Cheng Bao, Wolfgang G. Bessler, Journal of Power Sources 275, 922-934, (2015).

15. K. G. Nishanth, P.Sridhar, S. Pitchumani,and A. K. Shukla, Journal of The Electrochemical Society, 158 (8) B871-B876, (2011).

16. Rongzhong Jiang, Deryn Chu, Journal of The Electrochemical Society, 155,8, B798B803, (2008).

17. Rongzhong Jiang, Deryn Chu, Journal of The Electrochemical Society, 155,8,B804B810, (2008).

18. Sanjeev Mukerjee, Supramaniam Srinivasan, Manuel P. Soriaga, J.Electrochem. Soc., 142, 1409-1422, (1995).

19. Joseph R. Stetter, William R. Penrose, Sheng Yao Journal of The Electrochemical Society, 150,2 S11-S16, (2003).

20. Wenzhen Li, Qin Xin, Yushan Yan, International journal of Hydrogen energy, 35, 25302538, (2010). 